\title{
Multidimensional indices of achievements and poverty: what do we gain and what do we lose? An introduction to JOEI Forum on multidimensional poverty
}

\author{
Nora Lustig
}

Received: 3 May 2011 / Accepted: 17 May 2011 / Published online: 28 May 2011

(C) Springer Science+Business Media, LLC 2011

Poverty and wellbeing are multi-dimensional. Nobody questions that deprivations and achievements go beyond income. There is, however, sharp disagreement on whether the various dimensions of poverty and wellbeing can be aggregated into a single, multi-dimensional index in a meaningful way. Is aggregating dimensions of poverty and wellbeing useful? Is it sensible? These are the key questions underlying the three articles included in this issue of JOEI's Forum.

\section{The human development index}

One of the most popular and well-known multidimensional indices of achievements is UNDP's Human Development Index. In The HDI 2010: New Controversies, Old Critiques, Jeni Klugman (the director of the Human Development Report Office), Francisco Rodríguez and Hyung-Jin Choi state that "[t]he simplicity of the [Human Development] index's characterization of development (as an average of achievements in health, education and income), linked to the basic message that development is about much more than growth, has contributed to its popularity." From an advocacy perspective, the HDI has been useful and, judged by its presence in the media and political discourse, a resounding success. It has succeeded in deemphasizing a growth-centric view of development.

Throughout the years, scholars have been posing a number of criticisms of the HDI. Many of them have concentrated on two aspects: choice of dimensions and

\footnotetext{
N. Lustig $(\bowtie)$

Tulane University, New Orleans, LA, USA

e-mail:nlustig@tulane.edu

N. Lustig

Center for Global Development and Inter-American Dialogue, 1800 Massachusetts Avenue NW, Washington, DC, USA
} 
variables, and functional form. In terms of the functional form, the key concerns have related to the substitutability assumptions, the normalization of indicators, the asymmetric treatment of income, and the choice of weights. In 2010, the HDI experienced a significant makeover to address some of these criticisms. After a brief but comprehensive overview of the changes in the HDI since its introduction in 1990, Klugman et al. carefully explain the rationale behind the new HDI. This was the first time that major changes were introduced simultaneously to the indicators and functional form of the HDI.

The new HDI keeps the same three-dimensional structure with equal weights, but with several important changes. Life expectancy remains as the indicator for the health dimension; however, (log) Gross National Income replaces (log) GDP per capita as the measure for living standards, and mean years of schooling and expected years of schooling replace literacy and gross enrolment in the education dimension. The indices for each of the three dimensions are still normalized indicators of achievements but the practice of capping variables that surpass the upper bounds is eliminated. No additional dimensions are introduced into the new HDI primarily for pragmatic reasons. Measurement difficulties coupled with political controversies make the incorporation of dimensions such as indicators of agency, political freedoms, civil liberties and accountability very difficult. To address the key critique that the HDI neglected within-country inequality, three additional indices are introduced in the Human Development Report: the Inequality-Adjusted HDI, the Gender Inequality Index, and the Multidimensional Poverty Index.

Perhaps the most important modification introduced in 2010 is to replace the arithmetic mean by a geometric mean to aggregate the dimensions. This change "... marks a significant conceptual change in the way in which one conceives the relationship between different dimensions of capabilities." The old HDI functional form implied perfect substitutability. A geometric mean implies an elasticity of substitution between capabilities or dimensions equal to 1, thereby acknowledging the existence of diminishing marginal returns to increasing one capability while keeping others constant. A distinct advantage of the geometric mean, moreover, is that the rankings are invariant to the scale in which each variable is measured.

The HDI has also been criticized in more fundamental ways. There are those who consider it redundant: there is not much value added in aggregating income with nonincome dimensions to assess performance. By pinpointing the differences in rankings that emerge from a comparison between the HDI and per capita incomes, and the low correlation between income and non-income dimensions, Klugman et al. argue that adding non-income variables is not redundant.

Among the strongest criticism, however, is the one that considers the HDI misleading because it implies unappealing trade-offs between income and non-income variables. Ravallion [1], for example, has argued that the trade-offs implicit in the new HDI should be a cause of concern [1]. His calculations show that the marginal rate of substitution between longevity and income in the new HDI can vary from $\$ 0.53$ per year of life in Zimbabwe to about $\$ 9,000$ in the richest countries. For Ravallion, these tradeoffs have a policy implication that defeats common sense: to maximize human development in the world, the best would be to invest in higher life expectancy in rich countries. Klugman et al. retort by arguing that the HDI is an index of capabilities, conceptually different from a social welfare function. "The key difference is that a social welfare function is designed to be maximized, and thus 
the trade-offs along that social welfare function can be interpreted as values. But a capabilities index is meant to give a measure of the extent to which people in different countries have access to substantively different lives. Obviously, we care about the expansion of these capabilities and believe that expansion is welcome. But this is very different from claiming that the maximization of capabilities should be the only objective of social action." The HDI, the authors argue, is not meant to be used as a global social welfare function to be maximized. In turn, Ravallion responds that “... defining the MRS does not imply that the function concerned is a maximand in an optimization problem. The MRS is simply an interesting property of the function, telling us its relative weights at each data point." Ravallion argues elsewhere that "in the new HDI [a] poor country experiencing falling life expectancy due to (say) a collapse in its health-care system could still see its HDI improve with even a small rate of economic growth." "Would the trade-offs implicit in the HDI make it an inadequate index to measure development performance across countries and over time?

\section{Multidimensional poverty indices}

The interest in finding sensible synthetic multidimensional poverty indices has been around for quite some time. In the last decade, the quest has picked up pace, and measurement methodologies and their practical application have blossomed. This has also kindled the debate, as exemplified by Ravallion's paper included in this issue (and discussed below). Alkire and Foster's paper Understandings and Misunderstandings of Multidimensional Poverty Measurement is designed to clarify the potentials of multidimensional poverty measures (in particular, the AlkireFoster method) and to help distinguish misunderstandings from genuine limitations.

When poverty is conceived as multiple simultaneous deprivations, the question of identifying the poor ('who is poor?') takes on a different meaning. In the literature, some authors have suggested to use the union and others the intersection approach to identify the multi-dimensional poor. In real life, this leads to not very interesting or practical results (either too many or too few are classified as poor). In response, Alkire and Foster propose a method to generate multi-dimensional indices in which the union and the intersection are special cases. "The result is a methodology for measuring poverty in the sense of Sen (1976) that first identifies who is poor, then aggregates to obtain overall measures of poverty that reflect the multiple deprivations experienced by the poor." The multi-dimensional poverty measures derived from applying the $\mathrm{M}_{0}$ methodology—as the authors call it—fulfill desirable axioms, are decomposable, and include discrete and qualitative data as well as continuous and cardinal data.

The construction of multi-dimensional poverty measures using the $\mathrm{M}_{0}$ methodology involves the selection of dimensions, dimensional cutoffs (to determine when a person is deprived in a particular dimension), dimensional weights (to indicate the relative importance of the different deprivations), and a poverty cutoff (to determine when a person experiences enough deprivations to be considered poor). The essence

${ }^{1}$ Ibid. 
of identifying the poor in that multi-dimensional framework is to use a "dual cutoff" method. The "deprivation cutoff" is used to determine whether a person is poor in a particular dimension. It is analogous to the poverty line in the unidimensional analysis. The second cutoff is called the "poverty cutoff." It is the number of deprivations (or the weighted sum if unequal weights are used) that defines a person as poor. If the number is equal to 1 , the multi-dimensional index will be the union of deprivations. If it equals the number of dimensions, the index will be the intersection of deprivations. There exists an array of possibilities in between these two extremes. Once the poor are identified, the aggregation methodology follows a procedure analogous to the construction of FGT poverty measures in unidimensional analysis. Thus, the method yields adjusted headcount, poverty gap and squared poverty gap ratios.

Alkire and Foster emphasize that the choice of dimensions, weights and dual cut-offs is not pre-determined. Their "methodology is a general framework for measuring multidimensional poverty-an open source technology that can be freely altered by the user to best match the measure's context and evaluative purpose." Why should we be interested in an aggregation of the deprivations? According to Alkire and Foster, "[t]he value of having a poverty measure like $\mathrm{M}_{0}$ is that it can show change over time in a unified and internally consistent framework. This overall picture of change can be coherently and consistently deepened and sharpened by the more specific insights contained in decompositions and partial indices. Understanding this combination-an overview indicator that can give rise to a range of more specific analyses-is key to our measurement approach."

Since it was first published, the AF framework has faced a series of misunderstandings and critiques. Alkire and Foster attempt to address them. First, the authors clarify that AF measures are different from multi-dimensional marginal measures of poverty. The AF measures are sensitive to the joint distribution of deprivations, a characteristic that is absent from multi-dimensional marginal measures because these measures do not look across dimensions for the same individual. The value in marginal measures would be exactly the same if $x$ percent of people were simultaneously deprived in all dimensions, or if different slices of the population (where the slices are equal to the number of dimensions) were $\mathrm{x}$ percent deprived in just one of the dimensions in sequence. In the AK measures, the values would be different (except in the special case of the union). Another misunderstanding has arisen with respect to the type of data needed to estimate AK measures. Because each variable must be linked to a particular household, all variables must normally come from the same survey source; this is different from the marginal measures for which deprivations can be obtained from different sources. While the $\mathrm{M}_{0}$ methodology is specifically used by Alkire and Santos [2] to develop a Multidimensional Poverty Index (MPI), the AF methodology is flexible with respect to the dimensions, cutoffs, weights, and indicators used [2]. Alkire and Santos is just one application of the methodology. As stated by the authors, "multidimensional poverty measures can and often do take form in active relationship with participatory and deliberative processes about what poverty is and what current priorities might be. In particular, because the dimensions, indicators, weights and cutoffs of our methodology are flexible, they can be filled in many ways."

Alkire and Foster also explain that the foundational theoretical structure for the AF methods lies in the axiomatic approach. Their paper formulates and discusses the 
axioms that their methods satisfy. In this sense, AF methods (and derived measures) are subject to the same scrutiny applied to other indicators in the literature on axiomatic poverty measurement. The authors emphasize the importance of distinguishing between a measurement method and the elements used in its implementation. "There is a clear difference between evaluating the axiomatic characteristics of a measurement method and questioning the calibrations underlying a particular implementation of the approach. For example, there may be broad agreement on the use of the squared gap FGT measure, but disagreement over using income or consumption as the variable, or about the specific poverty line, or which PPP rates to use. In the multidimensional case, there may be disagreement over deprivation weights and cutoffs, even if the AF methodology is selected. Any critique that relies on a specific set of implementation choices is contingent on those choices and may not reveal much about the general methodology—which is more properly evaluated in axiomatic terms."

The authors address the critique made to their methods due to their lack of consistency with the basic tenets of welfare economics. This criticism has been more vocally and visibly expounded by Ravallion and will be discussed below. Alkire and Foster agree that ideally one would like to ground poverty measures in a social welfare framework. However, as the literature suggests, this is a very challenging task in practice. "[M] uch of the exercise of selecting a welfare function is by definition normative and has many degrees of freedom. There will likely be a multiplicity of acceptable functions, and even if a unique welfare function could be agreed upon there is no unique transformation from welfare function to poverty measure." If one could agree on a specific function to measure welfare, poverty could be measured with welfare as the aggregator and a welfare threshold as the goal. All our troubles would be solved. But, as Alkire and Foster remind us "[i]t is the absence of such an aggregator that led to the consideration of the multidimensional approach in the first place."

Alkire and Foster suggest that "[a]n alternative exercise might be to see whether the tradeoffs implied by a poverty measure are broadly consistent with some underlying notion of social welfare." The authors remind us how some of the most commonly used poverty measures such as the headcount and poverty gap ratios are at variance with some basic notions of welfare. The latter, however, have not led to discarding their use. One should probably exhibit similar levels of tolerance towards the multidimensional measures of poverty. At the same time, proponents of the multidimensional poverty indices should find ways to ground the tradeoffs implied by them in welfare theory and link them to economics more directly.

\section{The critique to multidimensional indices of poverty}

In On Multidimensional Indices of Poverty, Martin Ravallion presents his main criticisms to aggregating multiple deprivations into a composite (or what he denominates) "mashup index." Ravallion's criticisms are not addressed to the notion of poverty as deprivations in multiple dimensions. In fact, he argues that even the so-called "unidimensional" consumption-or income-based poverty measures are multi-dimensional. In the conventional measures of poverty, a myriad of 
items/dimensions (food, clothing, housing, and so on) are aggregated using market prices or —when the latter do not exist-appropriate substitutes.

Ravallion's main criticisms to the current multi-dimensional poverty measures are twofold. What is the usefulness of aggregating deprivations into a composite index if, for policy purposes, a disaggregation will be indispensable? For example, if a policymaker wants to address income and services deprivation and different geographic areas reveal different degrees of deprivation in each of the two dimensions, clearly the aggregate index will be insufficient to guide policy action. His main concern, however, is that aggregation may not only be futile but that aggregate indices can be problematic or misleading. Ravallion argues that the MPIs (whether the Alkire and Santos type or any of the variants based on the $\mathrm{M}_{0}$ methodology) can be misleading because of their implicit marginal rate of substitution across dimensions, a point to which I shall come back below. While Ravallion recognizes the value of including the joint distribution of multiple deprivations embedded in the Alkire and Foster's $\mathrm{M}_{0}$ methodology, he argues that this can be achieved using standard statistical analysis. Furthermore, the demanding nature of data requirements to construct the MPI (or its variants) will result in measures that, in fact, end up having fewer dimensions (in the area of living standard, for example) than the typical consumption-based (so-called) unidimensional indices. Ravallion argues that such is the case for the Alkire and Santos MPI because the six indicators used for the living standards dimension are but a subset of what is included in consumption- or incomebased poverty measures.

The core of Ravallion's critique, however, is addressed to the implicit marginal rates of substitution in the MPI based on the $\mathrm{M}_{0}$-family of measures. In the context of poverty measures, Ravallion develops his argument by distinguishing between two approaches to estimating an aggregate poverty index. The "attainment aggregation" calculates a composite index for aggregate consumption using prices (actual or imputed when market prices are missing) to be compared to a poverty line defined in the same space. The conventional measures using attainment aggregation are the headcount, poverty gap and squared poverty gap ratios. The second approach measures poverty in each of the dimensions separately and then aggregates the dimension-specific "deprivations" into a composite index. Ravallion calls this "deprivation aggregation." "The composite index is then a (typically) non-linear aggregation of the component dimensions of welfare, where the 'weights' depend on the properties of the component-specific poverty measures and the distribution of each component in the population." The two approaches will generally not yield the same measure even if the poverty lines are consistent (that is, if prices can be used, the sum of the cutoffs by dimension would be equal to the poverty line in the "attainment approach"). Ravallion illustrates this in Fig. 1. With a consistent poverty line, the attainment aggregation will identify as poor all the individuals "whose consumption of the two goods is within the triangle with vertices, $z / p_{1}, 0$ and $z / p_{2}$. The deprivation aggregation approach identifies instead some subset of those with $x_{1}<z_{1}$ and/or $x_{2}<z_{2}$ (the two unbounded rectangles of width $z_{1}$ and $z_{2}$ in Fig. 1). Without knowing the weights and data one cannot say which will give the larger count for who is deemed to be poor."

In the deprivation-aggregation approach “... the weights on deprivations are taken to be known and explicit, while the weights on attainments are implicit. The papers in this literature provide some elegant mathematical formulations of 
Fig. 1 Attainment versus deprivation aggregation

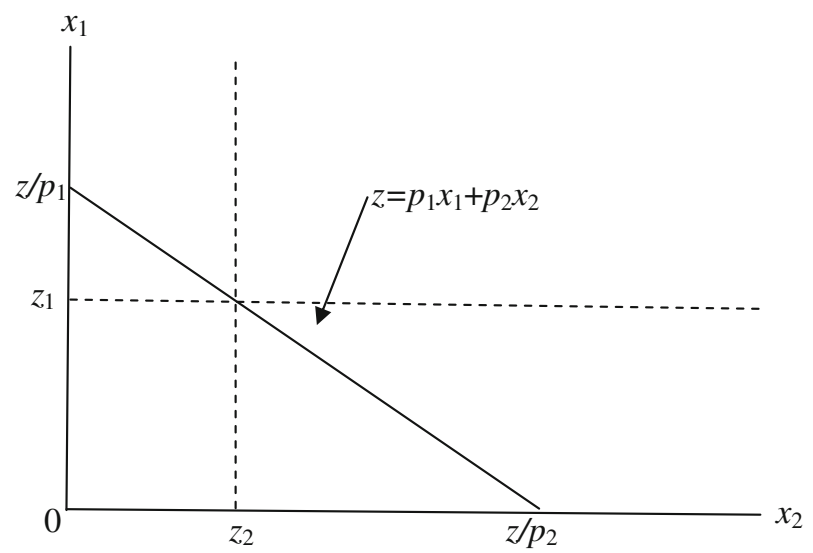

their measures, but provide little guidance for identifying the tradeoffs between attainments that are embodied in any measure based on this approach. The interest in identifying the tradeoffs built into a MIP does not rest on any view that it should be seen as a policy maximand. Rather the interest stems from the need to understand the properties of the index. The tradeoff is given by the marginal rate of substitution (MRS), which is simply the marginal weight on one attainment relative to that on another. However, the implied MRSs built into past MIPs have received little attention; indeed, the MRS is rarely even mentioned as a formal property."

The MRS facing someone at the poverty line in the attainment aggregation approach, is the relative price, $p_{1} / p_{2}$. "As long as the poverty bundle is consistent with the choices made by someone living at the poverty line, ... welfare consistency is assured with appropriate calibration." The MRS implicit in the deprivationaggregation approach are very different. They would only equal $p_{1} / p_{2}$ "by a fluke." Ravallion concludes "that aggregation across deprivations cannot in general yield poverty measures that are consistent with the welfare of someone living at the poverty line. This is because deprivation aggregation essentially ignores all implications for welfare measurement of consumer choice in a market economy. While those implications need not be decisive in welfare measurement, it is clearly worrying if the implicit tradeoff between any two market goods built into a poverty measure differs markedly from the tradeoff facing someone at the poverty line. When calibrated correctly, an attainment-aggregation measure guarantees that poor people would accept the tradeoffs built into the poverty measure. But there is no obvious calibration method for which this holds using deprivation aggregation." The deprivation-aggregation approach "... will identify some people as poor because they are lacking in one or more things that they can afford, but have no interest in acquiring. Poverty comparisons between people and over time (which are never easy) could be especially problematic."

Ravallion acknowledges that advocates of deprivation-aggregation will not be moved by these observations "since they reject prices as weights." Of the existing arguments, he considers the criticism that prices are missing (i.e., not all goods are market goods) or not reliable (i.e., market prices are distorted and do not reflect true social valuations; they are not shadow prices) as the most important. To this 
criticism Ravallion responds "[o]ne can agree that market prices do not accord with shadow prices in general without preferring to aggregate in the deprivation space, which rejects the use of all prices ... It is one thing to recognize that not all goods are market goods, or that there are market distortions, and quite another to ignore market prices when they are data. The case for deprivation aggregation cannot rest solely on the deficiencies of market prices as a means of valuation."

\section{The future}

At the bottom of the discussion is a fundamental disagreement on the "legitimacy" of the weights used to aggregate dimensions of wellbeing. The advocates of the deprivation-aggregation approach consider that prices are non-existent or are highly imperfect weights. Prices are the result of a "voting" system in the market that imbeds consumer preferences. However, markets are imperfect and market prices are unlikely to be equal to shadow prices. Even if they were, welfare theory shows that general-equilibrium prices will be influenced by the distribution of wealth. Given all the shortcomings of relative prices, the choice of weights should follow a voting system different from the market: for example, through participatory and deliberative processes about the nature of poverty and the selection of priorities. Advocates of the achievements-aggregation approach agree that prices may have shortcomings but that they convey information about trade-offs. Moreover, Ravallion and those who agree with him consider that the alternative weights used in the MPI (or similar indices) are not a good solution as they may imply unappealing trade-offs and that these aggregate poverty measures are generally not consistent with consumer welfare theory. In addition, Ravallion argues, there is little evidence that deliberation has influenced the choice of weights over the years. Thus, given this problem and the fact that for policy purposes disaggregation will be required, Ravallion asks: what is the advantage of using composite indices such as the HDI or the MPI instead of a "dashboard" of multiple indices? One key unresolved issue in the "dashboard approach," however, is that if we agree that welfare depends on a series of dimensions, how do we address the fact that the marginal effect of increasing an individual's access to one of the dimensions (e.g., health services) depends not only on that individual's access to the dimension in question, but also on the individual's level of all the other indicators of welfare?

Future research will need to focus on how to identify weights in ways that are consistent (1) with welfare economics and (2) with theories of justice. Will we have to choose between the two?

\section{References}

1. Ravallion, M.: Troubling tradeoffs in the human development index. World Bank, Washington DC, Policy Research Working Paper 5484 (2010)

2. Alkire, S., Santos, M.E.: Acute multidimensional poverty: a new index for developing countries. (OPHI working paper series, 38) (2010) 